\title{
Neural Network Identification and Sliding Mode Control for Hysteresis Nonlinear System with Backlash-Like Model
}

\author{
Ruiguo Liu (i) and Xuehui Gao (i) \\ Department of Mechanical and Electrical Engineering, Shandong University of Science and Technology, Taian 271019, China \\ Correspondence should be addressed to Xuehui Gao; xhgao@163.com
}

Received 14 March 2019; Revised 22 May 2019; Accepted 26 June 2019; Published 16 July 2019

Guest Editor: Jianwu Zeng

Copyright ( 2019 Ruiguo Liu and Xuehui Gao. This is an open access article distributed under the Creative Commons Attribution License, which permits unrestricted use, distribution, and reproduction in any medium, provided the original work is properly cited.

A new neural network sliding mode control (NNSMC) is proposed for backlash-like hysteresis nonlinear system in this paper. Firstly, only one neural network is designed to estimate the unknown system states and hysteresis section instead of multiscale neural network at former researches since that can save computation and simplify the controller design. Secondly, a new NNSMC is proposed for the hysteresis nonlinearity where it does not need tracking error transformation. Finally, the Lyapunov functions are adopted to guarantee the stabilities of the identification and control strategies semiglobally uniformly ultimately bounded (UUB). Two cases simulations are proved the effectiveness of the presented identification approach and the performance of the NNSMC.

\section{Introduction}

Many systems make hysteresis nonlinearities such as power systems, motor systems, and intelligent material systems. In some systems, the hysteresis can be applied to solve some problem. For example, some waveform generator systems utilized hysteresis to produce the designed waveform. But in most systems, the existence of the hysteresis nonlinearities will degrade the performance of the system and even lead to unstabilized in severe case. Therefore, the hysteresis investigations attract attention and also have many results for either system.

Due to the conflict of the fast development of economic and fossil energy lacking and pollution, it is inevitable to develop clean reproducible energy. Then, the new energy systems become more important and attract more attention than before. For example, the new energy vehicles will become the mainstream of the vehicle investigation and will occupy large scale market. But in most new energy systems, the existence of the hysteresis hinders the research progress and affects the improvement of the new energy application. Therefore, to investigate the hysteresis nonlinearity has important significance for new energy systems.

The hysteresis models have been investigated for many years. There are four familiar hysteresis models which are
Preisach model, Prandtl-Ishlinskii (P-I) model, Bouc-Wen model, and backlash-like model. These hysteresis models are all mathematical models that have more extensive application compared with the physical models such as Jiles-Atherton's (J-A) model. The Preisach model is the premier mathematical hysteresis model to deal with the magnetic systems firstly, but now it has a broader application for most hysteresis systems. We [1] proposed a novel identification to estimate the discrete Preisach model, where the new approach utilized the lower triangular matrix calculating the Preisach density function. The proposed identification could save computation and deduce complexity contrasting with the classic identification. We also investigated the Bouc-Wen hysteresis model of motor systems in [2]. The hysteresis motor servo system with BoucWen model firstly was transformed into a canonical state space form, then, a high-order neural network observer (HONN) was proposed and a filter for the tracking errors was adopted to simplify the controller design. Different from [2], we researched a backlash-like hysteresis system in [3]. A new Multiscale Chebyshev Neural Network (MCNN) was proposed to estimate the unknown system parameters and hysteresis section. Then the vector error was transformed into scalar error simplifying the controller and the stability of the closed system was guaranteed by Lyapunov function. In [3], two Chebyshev NN were applied to estimate the unknown 
states and hysteresis sections, respectively, and the tracking error was transformed to simplify the adaptive controller design. Nevertheless, we continue to investigate the backlashlike hysteresis model in this paper since it is fewer parameters and has analytical solution, but we will propose new $\mathrm{NN}$ and no longer use adaptive controller designing the controller for hysteresis systems.

Neural network is an important intelligent approach for identification and control systems [4-8]. Different NN structure was proposed and applied for different systems. A high-order neural network (HONN) was utilized to identification and control nonlinear system in $[9,10]$. A multitimescale recurrent high-order neural networks (MSRHONN) was proposed in [10] for singularly perturbed systems by the optimal bounded ellipsoid algorithm. The new approach could achieve faster convergence and the controller structure was simplified. In [3], the Multiscale Chebyshev Neural Network (MCNN) was presented to identification the hysteresis nonlinear systems. As an important intelligent method, The $\mathrm{NN}$ will be attended broader.

Different from adaptive control [11, 12], sliding mode control (SMC) [13] draws more attention in recent years. In some circuit, the hysteresis was utilized by the overshoot of the output voltage response. Literature [13] took advantage of the inherently variable-structure of the DC-DC system to design a modified sliding mode control regulating the DC hysteresis circuit system. The fuzzy adaptive ProportionalIntegral-Derivative (PID) SMC was proposed for hysteresis piezoactuated nanopositioning stage in [14]. Meanwhile, we also proposed SMC to control the hysteresis motor servo system with discrete Preisach model in [1]. A composite control consisting of discrete inverse model-based controller (DIMBC) and discrete adaptive sliding mode controller (DASMC) was presented for discrete hysteresis systems which could accelerate the reaching time and enhance the robustness of the controller. In this paper, we further utilize the SMC to deal with a continuous hysteresis system with Baclash-Like system. Since the existing of the chattering in SMC, the control performance will be degraded. If not handling it, when the chattering is serious, that will damage the equipment in real system. Several approaches can restrain the chattering. We have investigated the influence and the restrained method of the chattering in [1]. Then, in this paper, we adopt $\tanh (\cdot)$ function replacing the sign(.) function; for the $\tanh (\cdot)$ function is a continuous function, and it can well restrain the chattering of the SMC.

This research is a further investigation based on [3]. But it has three points different from [3]. First is one new $\mathrm{NN}$ designed to estimate the unknown states and hysteresis sections in this research which is different from two Multiscale Chebyshev Neural Networks (MSCNN) in [3] since the new design will simplify the controller and reduce the computation. We adopt one $\mathrm{NN}$ in this research because almost new energy systems only have one time scale, it does not need multiscale NN structure. But beyond that, the investigations of new energy system with NN controller are not common yet, one scale NN controller is researching, and multiscale NN will be investigate as well as other systems. Second is no tracking error transformation in this paper since the tracking error transformation in [3] could lead to the decline control performance. In this research, we transform the system into canonical form to avoid the complicated computation of controller design such as backstepping. Since the system is converted, the tracking error transformation is not necessary to simplify the computation. After all, the tracking error transformation is exactly save the calculation but can degrade the control precision. Finally, in this paper, we design a sliding mode control (SMC) instead of the adaptive control in [3]. The SMC controller has more strong robustness which can preferably compensate the error and disturbance result from the $\mathrm{NN}$ and other external causes.

The rest of the paper is organised as follows: Section 2 states the problem formulation. The NN identification design is proposed in Section 3 and Section 4 shows SMC controller design. The simulations are given in Section 5. Section 6 concludes the papers. Data availability statement, conflicts of interest and acknowledgments are at the end of the paper.

\section{Preliminaries}

Considering a hysteresis nonlinear system is described as

$$
\begin{aligned}
\dot{x} & =f_{1}(x)+f_{2}(x) v \\
\dot{v} & =g(x, u) u
\end{aligned}
$$

with $f_{i}(0)=0, i=1,2$ and $g(0,0)=0$, where $x \in \mathbb{R}^{n}, v \in$ $\mathbb{R}^{m}$ are the states and intermediate input, respectively, $u \in \mathbb{R}^{l}$ is the control input, $f_{i}, g, i=1,2$ denote unknown general nonlinear smooth functions, respectively, and $v$ represents hysteresis nonlinearity. In this paper, we discuss the hysteresis nonlinearity $v$ that is described by backlash-like model. The backlash-like hysteresis $v$ can be expressed as follows [15]:

$$
\dot{v}=g(x, u) u=\gamma|\dot{u}|(\alpha u-v)+\beta \dot{u},
$$

where $\gamma>0, \alpha>0$ are positive constants and $\alpha>\beta$.

The figure of backlash-like hysteresis is illustrated in Figure 1, where $\alpha=2.3, \beta=0.15, \gamma=1.1$, and the input $u=K \sin (6 t), K_{1}=4, K_{2}=3$, and $K_{3}=2$.

To address the unknown system state function $f_{1}(x)$, $f_{2}(x)$, system (1) are rewritten as follows:

$$
\begin{aligned}
\dot{x} & =A x+\bar{f}_{1}(x)+B v+\bar{f}_{2}(x) \\
\dot{v} & =g(x, u) u,
\end{aligned}
$$

where $A \in \mathbb{R}^{n \times n}$ is Hurwitz matrix, $B \in \mathbb{R}^{n \times 1}, A, B$ are controllable, and $\bar{f}_{1}(x)=f_{1}(x)-A x, \bar{f}_{2}(x)=f_{2}(x)-B$.

Considering the Hurwitz state matrix $A$, given a positive definite matrix $P=P^{T}>0$, there exists positive definite matrix $Q=Q^{T}>0$ such that the inequality

$$
A^{T} P+P A \leq-Q
$$

holds. 


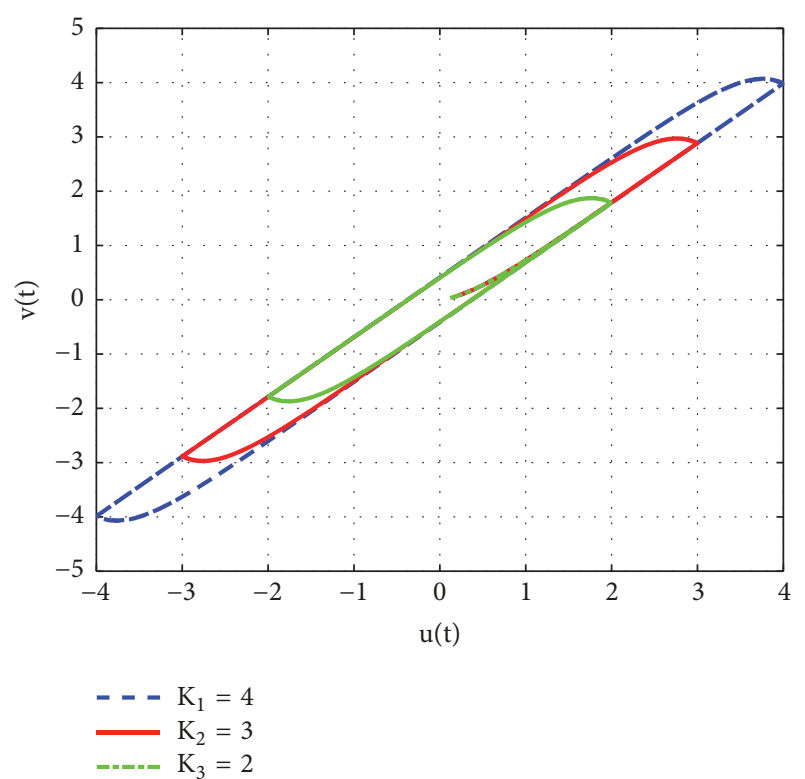

FIGURE 1: The curves of the backlash-like hysteresis models.

According to $[3,16]$, the equation $\dot{v}=g(x, u) u$ can be solved as

$$
\begin{aligned}
v= & \alpha u+\left(v_{0}-\alpha u_{0}\right) e^{-\gamma\left(u-u_{0}\right) \operatorname{sign}(\dot{u})} \\
& +e^{-\gamma u \operatorname{sign}(\dot{u})} \int_{u_{0}}^{u}(\beta-\alpha) e^{\gamma \xi \operatorname{sign}(\dot{u})} d \xi
\end{aligned}
$$

where $v_{0}, u_{0}$ are the initial values of $v, u$.

Then, substituting the backlash-like model (2) into system (3), we have

$$
\begin{aligned}
\dot{x}= & A x+\bar{f}_{1}(x)+B \alpha u+B\left(v_{0}-\alpha u_{0}\right) e^{-\gamma\left(u-u_{0}\right) \operatorname{sign}(\dot{u})} \\
& \left.+B e^{-\gamma u \operatorname{sign}(\dot{u})} \int_{u_{0}}^{u}(\beta-\alpha) e^{\gamma \xi \operatorname{sign}(\dot{u})} d \xi\right)+\bar{f}_{2}(x)
\end{aligned}
$$

Define a smooth function as follows:

$$
F(x)=\bar{f}_{1}(x)+\bar{f}_{2}(x),
$$

then system (6) can be deduced as

$$
\begin{aligned}
\dot{x}= & A x+B u+F(x)+B\left(v_{0}-\alpha u_{0}\right) e^{-\gamma\left(u-u_{0}\right) \operatorname{sign}(\dot{u})} \\
& \left.+B e^{-\gamma u \operatorname{sign}(\dot{u})} \int_{u_{0}}^{u}(\beta-\alpha) e^{\gamma \xi \operatorname{sign}(\dot{u})} d \xi\right) .
\end{aligned}
$$

\section{NN Identification Design}

Neural network (NN) can commendably approximate the nonlinearity systems. But different NN structure has different characteristics. In this paper, we adopt high-order neural network (HONN) to estimate the unknown general nonlinear smooth functions and hysteresis nonlinearity. The HONN is an excellent NN for nonlinear systems, where the big advantage is that only needs less neurons and computational costs. Thus the HONN developed high-order basis function connections so that it can easily approximate the high-order nonlinearities.

Considering system (8), the unknown function $F(x)$ and the hysteresis nonlinearity $B\left(v_{0}-\alpha u_{0}\right) e^{-\gamma\left(u-u_{0}\right) \operatorname{sign}(\dot{u})}+$ $\left.B e^{-\gamma u \operatorname{sign}(\dot{u})} \int_{u_{0}}^{u}(\beta-\alpha) e^{\gamma \xi \operatorname{sign}(\dot{u})} d \xi\right)$ will be approximated by NN. Hence there exists unknown bounded NN weight matrix $W \in \mathbb{R}^{n \times p}$ and $\Psi(x)=\left[\Psi_{1}, \Psi_{2}, \ldots, \Psi_{p}\right]^{T} \in \mathbb{R}^{p}$ which can be identified by the following model:

$$
\dot{\hat{x}}=A \hat{x}+B u+W \Psi,
$$

where the activation function $\Psi(x)$ is defined as

$$
\begin{aligned}
\Psi(x) & =\left[\Psi_{1}, \Psi_{2}, \ldots, \Psi_{p}\right]^{T} \in \mathbb{R}^{p} \\
\Psi_{k} & =\prod_{i \in L}[\psi(x)]^{d_{i}(k)}, \quad k=1,2, \ldots, p \\
\psi(x) & =\frac{c}{1+e^{-d x}}+h,
\end{aligned}
$$

where $L$ represent the collections of $p$ node ordered subsets, $c, d, h$ are designed parameters, and $d_{i}(k)$ is designed nonnegative integer.

Assuming an ideal NN can be approximated system (1) as

$$
\dot{x}=A x+B u+W^{*} \Psi+\varepsilon,
$$

where $W^{*}$ means the ideal NN weight matrix, the $\mathrm{NN} W^{*} \Psi$ completely substitutes the unknown function $F(x)$ and the hysteresis nonlinearity, $\varepsilon$ is a bounded approximation error, i.e., $\|\varepsilon\| \leq \varepsilon_{M}$, and $\varepsilon_{M}$ is a known positive constant vector.

Define the identification error $e$ as

$$
e=x-\widehat{x}
$$

Then, the derivative of the error $e$ can be deduced by (9) and (11):

$$
\dot{e}=\dot{x}-\dot{\hat{x}}=A e+\widetilde{W} \Psi+\varepsilon,
$$

where $\widetilde{W}=W^{*}-W$.

The identification structure of HONN can be illustrated in Figure 2.

Considering references $[9,17]$, the following assumption is proposed.

Assumption 1. The error norm of $\mathrm{NN}$ weight matrix $\widetilde{W}$ is bounded by $\|\widetilde{W}\| \leq W_{M}$, and the basis function of $\mathrm{NN}$ is bounded, i.e., $\|\Psi\| \leq \psi_{M}$.

Theorem 2. Considering system (1) which is approximated by the NN of (11), if the identification error is defined as (12), based on the Assumption 1, the identification e is semiglobally uniformly ultimately bounded (UUB) and it converges to a small compact set around zero as $\|e\| \leq \sqrt{2\left(\eta_{2} / \eta_{1}\right) /\|P\|}$, where $\eta_{1}, \eta_{2}$ are defined in (17). 


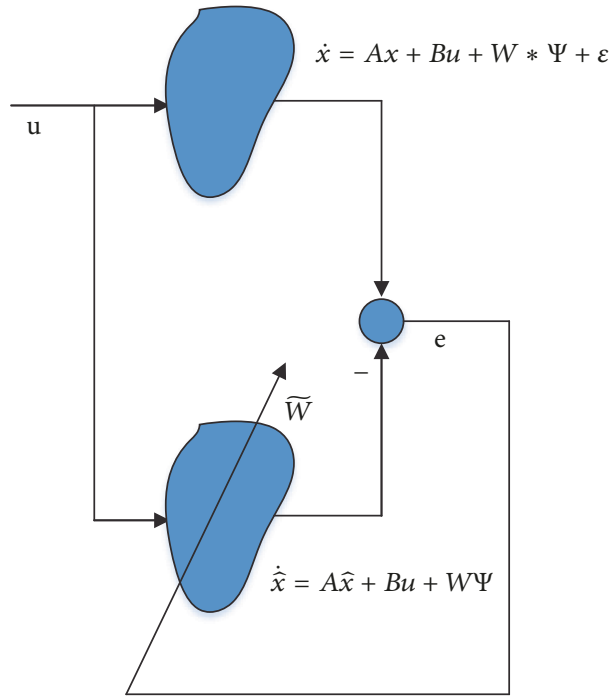

FIgURE 2: The structure of the NN identification.

Proof. Define the Lyapunov function as

$$
V=\frac{1}{2} e^{T} P e
$$

From (13), the derivative of $V$ can be deduced as

$$
\begin{aligned}
\dot{V}= & \frac{1}{2} \dot{e}^{T} P e+\frac{1}{2} e^{T} P \dot{e} \\
= & \frac{1}{2}(A e+\widetilde{W} \Psi+\varepsilon)^{T} P e+\frac{1}{2} e^{T} P(A e+\widetilde{W} \Psi+\varepsilon) \\
= & \frac{1}{2} e^{T} A^{T} P e+\frac{1}{2} \Psi^{T} \widetilde{W}^{T} P e+\frac{1}{2} \varepsilon^{T} P e+\frac{1}{2} e^{T} P A e \\
& +\frac{1}{2} e^{T} P \widetilde{W} \Psi+\frac{1}{2} e^{T} P \varepsilon \\
\leq & -\frac{1}{2} e^{T} Q e+e^{T} P(\widetilde{W} \Psi+\varepsilon) \\
\leq & -\frac{1}{2} e^{T} Q e+\left\|e^{T}\right\|\|P\|(\|\widetilde{W} \Psi\|+\|\varepsilon\|) .
\end{aligned}
$$

Invoking Young's inequality $a b \leq\left(a^{2}+b^{2}\right) / 2$ on the term $\left\|e^{T}\right\|\|P\|(\|\widetilde{W} \Psi\|+\|\varepsilon\|)$, we have

$$
\begin{aligned}
& \left\|e^{T}\right\|\|P\|(\|\widetilde{W} \Psi\|+\|\varepsilon\|) \\
& \leq \frac{\left\|e^{T}\right\|^{2}\|P\|^{2}+(\|\widetilde{W} \Psi\|+\|\varepsilon\|)^{2}}{2},
\end{aligned}
$$

then considering Assumption 1 and (16), the derivative of $V$ in (15) yields

$$
\begin{aligned}
\dot{V} & \leq-\frac{1}{2} e^{T} Q e+\frac{\left\|e^{T}\right\|^{2}\|P\|^{2}+\left(W_{M} \psi_{M}+\varepsilon_{M}\right)^{2}}{2} \\
& \leq-\eta_{1} V+\eta_{2}
\end{aligned}
$$

where $\eta_{1}=\left((1 / 2) e^{T} Q e+\left\|e^{T}\right\|^{2}\|P\|^{2} / 2\right) / e^{T} P e ; \eta_{2}=\left(W_{M} \psi_{M}+\right.$ $\left.\varepsilon_{M}\right)^{2} / 2$.

According to the Lyapunov theorem, $V$ is UUB and the identification error $e$ is bounded. Moreover, integrating both sides of (17) over the time [0.t], the inequality can be solved as

$$
V \leq V_{0} e^{-\eta_{1} t}+\frac{\eta_{2}}{\eta_{1}}\left(1-e^{-\eta_{1} t}\right) \leq \frac{\eta_{2}}{\eta_{1}}+V_{0} e^{-\eta_{1} t}
$$

Then, by applying (14), the bound of the identification error $e$ can be solved as

$$
\|e\| \leq \sqrt{\frac{2\left(\eta_{2} / \eta_{1}+V_{0} e^{-\eta_{1} t}\right)}{\|P\|}}
$$

such that it has

$$
\lim _{t \rightarrow \infty}\|e\| \leq \lim _{t \rightarrow \infty} \sqrt{\frac{2\left(\eta_{2} / \eta_{1}+V_{0} e^{-\eta_{1} t}\right)}{\|P\|}}=\sqrt{\frac{2\left(\eta_{2} / \eta_{1}\right)}{\|P\|}} .
$$

That means the identification error $e$ can be converged to a small compact set around zero.

The proof is completed.

\section{Controller Design}

Considering system (1), if the system is a pure feedback system, in order to avoid the complicated computation of the back-stepping controller, new state variables are defined to transform the state variables into canonical form based on reference [9]. Therefore, the new alternative states can be defined as

$$
\begin{aligned}
& z_{1}=x_{1} \\
& z_{2}=\dot{z}_{1} \\
& z_{i}=\dot{z}_{i-1}, \quad i=2,3, \ldots, n,
\end{aligned}
$$

and the transformed canonical system is

$$
\begin{aligned}
& \dot{z}_{1}=z_{2} \\
& \dot{z}_{i}=z_{i+1}, \quad i=1,2, \ldots, n-1 \\
& \dot{z}_{n}=\dot{a}_{n}\left(x_{n}\right)+\dot{b}_{n} u,
\end{aligned}
$$

where the definition of $\dot{a}_{n}, \hat{b}_{n}$ can be obtained by the method of the reference [9].

For the existing of the hysteresis nonlinearity, we also can adopt the approach of reference [9] to avoid the complicated controller design. Considering $A$ is Hurwitz matrix and $A, B$ are controllable, without loss of generality, we can transform 
the system (8) into a normalized form. Then, assume the matrixes $A, B$ are

$$
\begin{aligned}
& A=\left[\begin{array}{cccc}
a_{11} & a_{12} & \cdots & a_{1 n} \\
a_{21} & a_{22} & \cdots & a_{2 n} \\
\vdots & \vdots & \ddots & \vdots \\
a_{n 1} & a_{n 2} & \cdots & a_{n n}
\end{array}\right], \\
& B=\left[\begin{array}{c}
b_{1} \\
b_{2} \\
\vdots \\
b_{n}
\end{array}\right],
\end{aligned}
$$

According to the fact that $z_{2}=r_{1}(x)=\dot{z}_{1}=a_{11} x_{1}+$ $a_{12} x_{2}+\cdots+a_{1 n} x_{n}+b_{1} u+\bar{f}_{1}(x(1))+\bar{f}_{2}(x(1))+b_{1}\left(v_{0}-\right.$ $\left.\left.\alpha u_{0}\right) e^{-\gamma\left(u-u_{0}\right) \operatorname{sign}(\dot{u})}+b_{1} e^{-\gamma u \operatorname{sign}(\dot{u})} \int_{u_{0}}^{u}(\beta-\alpha) e^{\gamma \xi \operatorname{sign}(\dot{u})} d \xi\right)$, it has the following:

$$
\begin{aligned}
\dot{z}_{2}= & \ddot{z}_{1}=\frac{\partial r_{1}(x)}{\partial x_{1}} \dot{x}_{1}+\frac{\partial r_{1}(x)}{\partial x_{2}} \dot{x}_{2} \\
= & a_{11} \dot{x}_{1}+a_{12} \dot{x}_{2}+\frac{\partial \bar{f}_{1}\left(x_{1}\right)}{\partial x_{1}} \dot{x}_{1}+\frac{\partial \bar{f}_{2}\left(x_{1}\right)}{\partial x_{1}} \dot{x}_{1} \\
& +\frac{\partial \bar{f}_{1}\left(x_{1}\right)}{\partial x_{2}} \dot{x}_{2}+\frac{\partial \bar{f}_{2}\left(x_{1}\right)}{\partial x_{2}} \dot{x}_{2} .
\end{aligned}
$$

Then, we also can obtain

$$
\begin{aligned}
\dot{z}_{3}=\ddot{z}_{2} & a_{12} \ddot{x}_{2}+\frac{\partial\left(\partial \bar{f}_{1}\left(x_{1}\right) / \partial x_{1}\right) \dot{x}_{1}}{\partial x_{2}} \dot{x}_{2} \\
& +\frac{\partial\left(\partial \bar{f}_{2}\left(x_{1}\right) / \partial x_{1}\right) \dot{x}_{1}}{\partial x_{2}} \dot{x}_{2} \\
& +\frac{\partial\left(\partial \bar{f}_{1}\left(x_{1}\right) / \partial x_{2}\right) \dot{x}_{2}}{\partial x_{2}} \dot{x}_{2} \\
& +\frac{\partial\left(\partial \bar{f}_{2}\left(x_{1}\right) / \partial x_{2}\right) \dot{x}_{2}}{\partial x_{2}} \dot{x}_{2} \\
& +\frac{\partial\left(\partial \bar{f}_{1}\left(x_{1}\right) / \partial x_{1}\right) \dot{x}_{1}}{\partial x_{3}} \dot{x}_{3} \\
& +\frac{\partial\left(\partial \bar{f}_{2}\left(x_{1}\right) / \partial x_{1}\right) \dot{x}_{1}}{\partial x_{3}} \dot{x}_{3}
\end{aligned}
$$

$$
\begin{gathered}
+\frac{\partial\left(\partial \bar{f}_{1}\left(x_{1}\right) / \partial x_{2}\right) \dot{x}_{2}}{\partial x_{3}} \dot{x}_{3} \\
+\frac{\partial\left(\partial \bar{f}_{2}\left(x_{1}\right) / \partial x_{2}\right) \dot{x}_{2}}{\partial x_{3}} \dot{x}_{3} .
\end{gathered}
$$

By parity of reasoning, we apply the method of reference [9], the canonical form of system (8) can be obtained as (22).

According to the preceding discuss, without loss of generality, the coefficient matrixes $A, B$ can be adopted as

$$
\begin{aligned}
A & =\left[\begin{array}{ccccc}
0 & 1 & 0 & \cdots & 0 \\
0 & 0 & 1 & \cdots & 0 \\
0 & 0 & 0 & \cdots & 0 \\
\vdots & \vdots & \vdots & \ddots & \vdots \\
-a_{1} & -a_{2} & -a_{3} & \cdots & -a_{n}
\end{array}\right], \\
B & =\left[\begin{array}{c}
0 \\
0 \\
0 \\
\vdots \\
b
\end{array}\right] .
\end{aligned}
$$

Then, the system is described by NN in (11) which can be rewritten as

$$
\begin{aligned}
& \dot{x}_{1}=x_{2} \\
& \dot{x}_{2}=x_{3}
\end{aligned}
$$

$$
\dot{x}_{n}=-a_{1} x_{1}-a_{2} x_{2}-\cdots-a_{n} x_{n}+b u+C W^{*} \Psi+C \varepsilon,
$$

where $C \in \mathbb{R}^{1 \times n}$ is a transformed vector parameters for the $\mathrm{NN}$ in (11).

Remark 3. For the existing of the hysteresis nonlinearity and the nonlinear smooth functions $\left.\bar{f}_{(} x\right), \bar{f}_{2}(x)$, we cannot use the linear system method to transform the coefficient matrixes (23) into normalized form (26). But under some conditions, we also use the linear system method to transform the coefficient matrixes, that can be simplify the computation and is convenient for the controller design.

\section{Define the Sliding Mode Manifold $s$ as Follows}

$$
s=c_{1} e_{1}+c_{2} \dot{e}_{1}+\cdots+e_{1}^{(n)},
$$

where the error $e_{1}$ is defined as

$$
e_{1}=x_{1}-x_{d},
$$

where $x_{d}$ is the reference input signals. 
Design the sliding mode controller input as follows:

$$
u=\frac{1}{b}\left(-\varsigma \widehat{s}-C \widehat{W} \Psi-\widehat{m}+\widehat{z}+x_{d}^{(n+1)}-\operatorname{sign}(\widehat{s})\right)
$$

where $\varsigma>0, \widehat{z}=a_{1} \widehat{x}_{1}+a_{2} \widehat{x}_{2}+\cdots, a_{n} \widehat{x}_{n}, \widehat{m}=c_{1} \dot{\hat{e}}_{1}-c_{2} \ddot{\widehat{e}}_{1}-$ $\cdots-c_{n-1} \hat{e}_{1}^{(n-1)}$, and $\widehat{s}=c_{1} \hat{e}_{1}+c_{2} \dot{\hat{e}}_{1}+\cdots+\hat{e}_{1}^{(n)}$.

The NN update law is selected as

$$
\dot{\widehat{W}}=-\rho s \Psi
$$

where $\rho$ is designed parameter.

Define the errors as

$$
\tilde{x}_{i}=x_{i}-\widehat{x}_{i}, \quad i=1,2, \ldots, n .
$$

Then, the following theorem holds.

Theorem 4. Considering system (27), the sliding mode manifold is defined in (28), the controller is chosen as in (30), the NN update law is chosen as in (31), and then, the control system is semiglobally uniformly ultimately bounded (UUB).

Proof. Define the Lyapunov function as

$$
V_{t}=\frac{1}{2} s^{2}
$$

According to (28), (29), and (30), we have

$$
\begin{aligned}
\dot{V}_{t} & =s \dot{s}=s\left(c_{1} \dot{e}_{1}+c_{2} \ddot{e}_{1}+\cdots+e_{1}^{(n+1)}\right)=s\left(c_{1} \dot{e}_{1}+c_{2} \ddot{e}_{1}\right. \\
& +\cdots-a_{1} x_{1}-a_{2} x_{2}-\cdots-a_{n} x_{n}+b u+C W^{*} \Psi \\
& \left.+C \varepsilon-x_{d}^{(n+1)}\right)=s\left(c_{1} \dot{e}_{1}+c_{2} \ddot{e}_{1}+\cdots-a_{1} x_{1}-a_{2} x_{2}\right. \\
& -\cdots-a_{n} x_{n}+C W^{*} \Psi+C \varepsilon-\varsigma \widehat{s}-\widehat{m}+\widehat{z} \\
& -\operatorname{sign}(\widehat{s}))=s\left(-\varsigma \widehat{s}-a_{1} \widetilde{x}_{1}+\left(c_{1}-a_{2}\right) \tilde{x}_{2}+\cdots\right. \\
& \left.+\left(c_{n-1}-a_{n}\right) \tilde{x}_{n}+C \widetilde{W} \Psi+C \varepsilon-\operatorname{sign}(\widehat{s})\right)=-\varsigma s^{2} \\
& +s\left(-a_{1} \widetilde{x}_{1}+\left(c_{1}-a_{2}\right) \widetilde{x}_{2}+\cdots+\left(c_{n-1}-a_{n}\right) \widetilde{x}_{n}\right. \\
& +C \widetilde{W} \Psi+C \varepsilon+\varsigma \widetilde{s}-\operatorname{sign}(\widehat{s}))=-\varsigma s^{2} \\
& +s\left(\left(c_{1}-a_{1}\right) \tilde{x}_{1}+\left(c_{1}+c_{2}-a_{2}\right) \tilde{x}_{2}+\cdots\right. \\
& \left.+\left(c_{n-1}-a_{n}+1\right) \widetilde{x}_{n}+C \widetilde{W} \Psi+C \varepsilon-\operatorname{sign}(\widehat{s})\right)
\end{aligned}
$$

where $\widetilde{s}=s-\widehat{s}=c_{1} \widetilde{x}_{1}+c_{2} \tilde{x}_{2}+\cdots+\tilde{x}_{n}$.

Define $\Delta_{\text {max }} \geq \mid\left(c_{1}-a_{1}\right) \tilde{x}_{1}+\left(c_{1}+c_{2}-a_{2}\right) \tilde{x}_{2}+\cdots+\left(c_{n-1}-a_{n}+\right.$ 1) $\widetilde{x}_{n}+C \widetilde{W} \Psi+C \varepsilon \mid-\operatorname{sign}(\widehat{s})$, according to Young's inequality, (34) can be deduced as

$$
\dot{V}_{t} \leq-\varsigma s^{2}+\frac{1}{2}\left(s^{2}+\Delta_{\max }^{2}\right)=-(2 \varsigma-1) V_{t}+\frac{1}{2} \Delta_{\max }^{2} .
$$

To solve inequality (35), we have

$$
\begin{aligned}
V_{t} & \leq e^{-\varrho\left(t-t_{0}\right)} V\left(t_{0}\right)+\frac{1}{2} \Delta_{\max }^{2} \int_{t_{0}}^{t} e^{-\varrho(t-\xi)} d \xi \\
& =e^{-\varrho\left(t-t_{0}\right)} V\left(t_{0}\right)-\frac{1}{2 \varrho} \Delta_{\max }^{2}\left(1-e^{-\varrho\left(t-t_{0}\right)}\right),
\end{aligned}
$$

where $\varrho=2 \varsigma-1$.
Let the parameter $\varsigma>1 / 2$, and one can obtain

$$
\lim _{t \rightarrow \infty} V_{t} \leq \frac{1}{2 \varrho} \Delta_{\max }^{2}
$$

Therefore, based on the Lyapunov theory, the controlled system is semiglobally uniformly ultimately bounded (UUB).

The proof is completed.

Remark 5. Based on Theorem 4, all the closed loop signals are bounded. But on the structure of the NNSMC, we obviously known that the NN identification is a part of the closed loop and the update law of $\mathrm{NN}$ is deduced by the closed loop. Therefore, the identification connected with the control design, it is online estimating the nonlinear functions and online adjusting by the tracking errors.

\section{Simulations}

Considering a hysteresis system as follows:

$$
\begin{aligned}
\dot{x}_{1}= & x_{2} \\
\dot{x}_{2}= & \frac{1.3}{0.186} x_{1}+\frac{\cos x_{2}}{0.186} \\
& +\frac{2}{1+\sin \left(-0.5\left(x_{1}-x_{2}\right)\right)+0.12 \cos x_{2}} v \\
\dot{v}= & 0.08|\dot{u}|(2 u-v)+0.03 \dot{u} .
\end{aligned}
$$

6.1. Controller Design. In order to verify the effectiveness of the proposed NNSMC, A PID controller is designed to compare with the proposed NNSMC. The PID controller gains are chosen as $K P=50, K I=3, K D=1.26$. Otherwise, for NNSMC, the sliding mode manifold is defined as in (28), the NNSMC controller is defined as in (30). The parameters are selected as $c=6.28, d=2, h=1, c_{1}=5.95, a_{1}=6.99$, $a_{2}=5.38, b=5.38, \rho=0.05$, and $\varsigma=2$.

We design two different control input signal to test the proposed approaches. Firstly, a square wave signal is selected for the hysteresis system, and then two amplitude sine signals are adopted with NNSMC which will compare with the PID controller.

The two different controllers will test the system (38) with reference input. The proposed NNSMC firstly estimates the unknown states and hysteresis part with $\mathrm{NN}$ and uses the sliding mode controller to control the hysteresis system online.

6.2. Control Results. Two different reference input signals are selected to test the proposed NNSMC. The NN identification is adopted estimating the unknown nonlinear functions and then the SMC is applied to control the closed loop. In order to verify the effectiveness of the NN identification and SMC, A square wave signal firstly utilizes tracking the system. Two amplitude sine signals are adopted with NNSMC and PID controller. The details are listed as follows. 

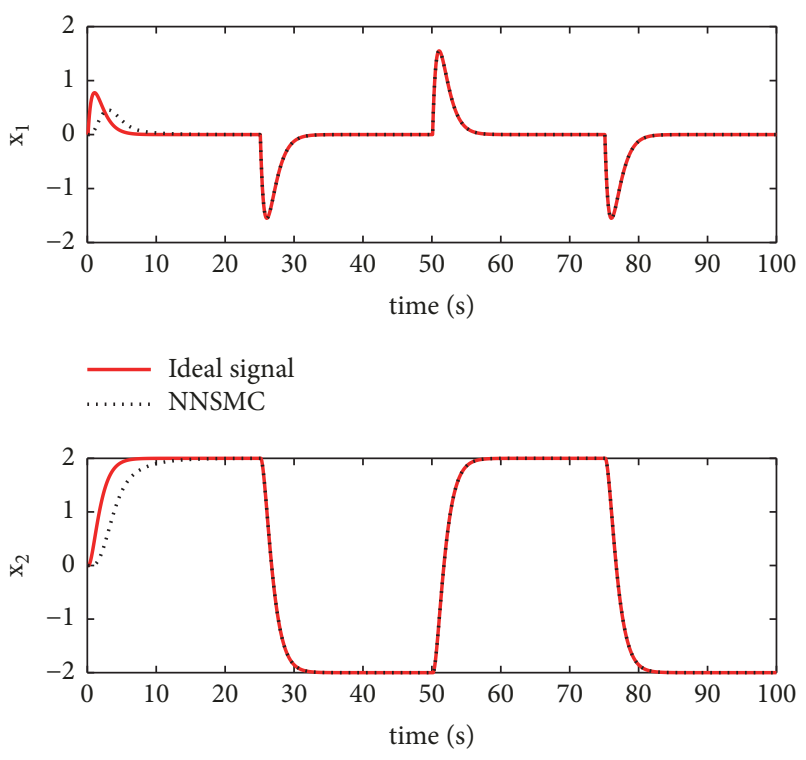

Ideal signal NNSMC

FIGURE 3: The control results of NNSMC versus PID control.
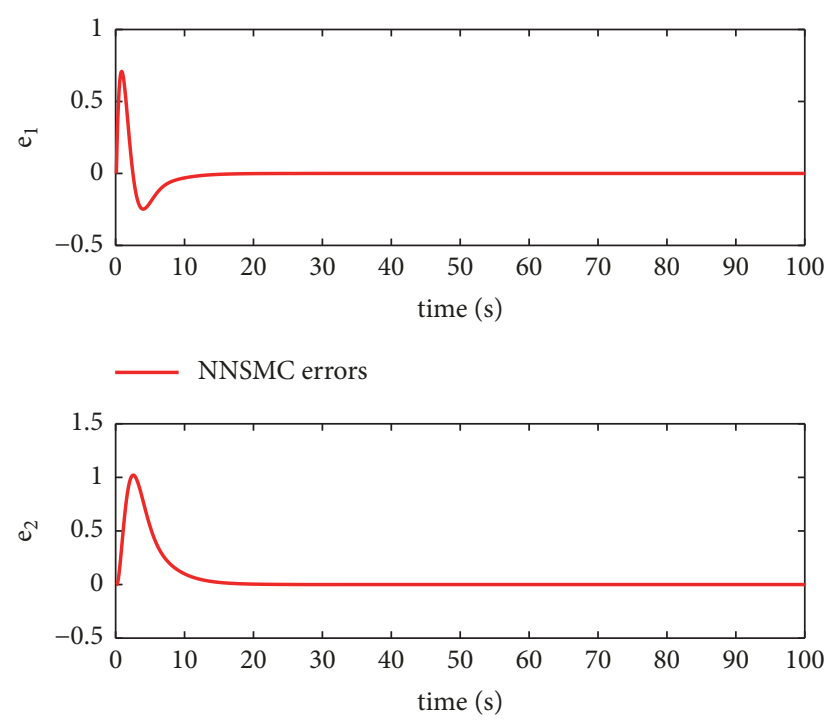

NNSMC errors

Figure 4: The control results of NNSMC versus PID control.

6.2.1. Case 1. We chose the reference square wave with amplitude which is $a=2$, and the duty ratio is $50 \%$. Then the tracking results are illustrated in Figure 3, the errors are shown in Figure 4, and the sliding mode reaching phase process is illustrated in Figure 5. From Figures 3 and 4, it is clear that the proposed NN identification and the NNSMC have a good tracking performance. The dynamic procedures are less than $10 \mathrm{~s}$ and the errors are shown in the same phenomenon.

In order to restrain chattering of the SMC, we adopt $\tanh (\cdot)$ function instead of the $\operatorname{sign}(\cdot)$ function in the simulations. From Figure 5, we can see that the chattering is

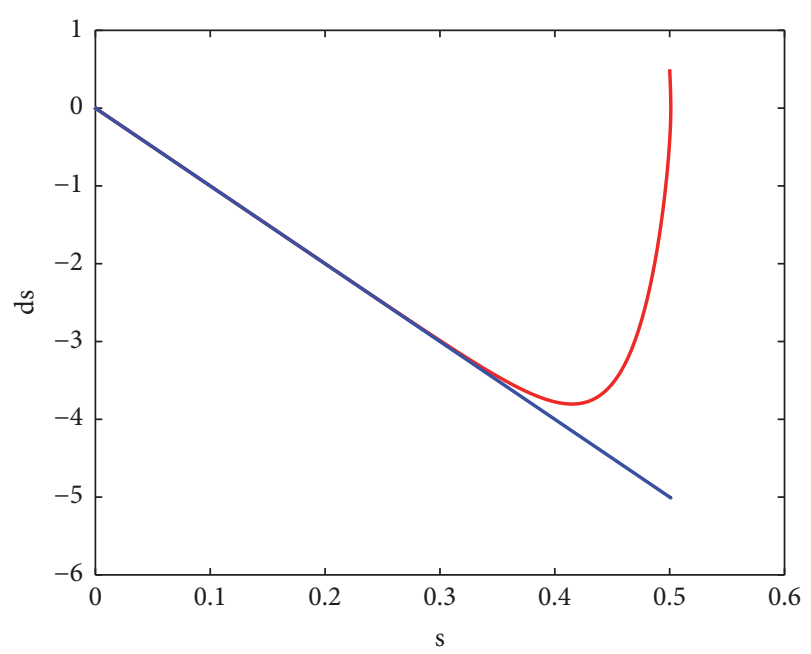

FIGURE 5: The control results of NNSMC versus PID control.
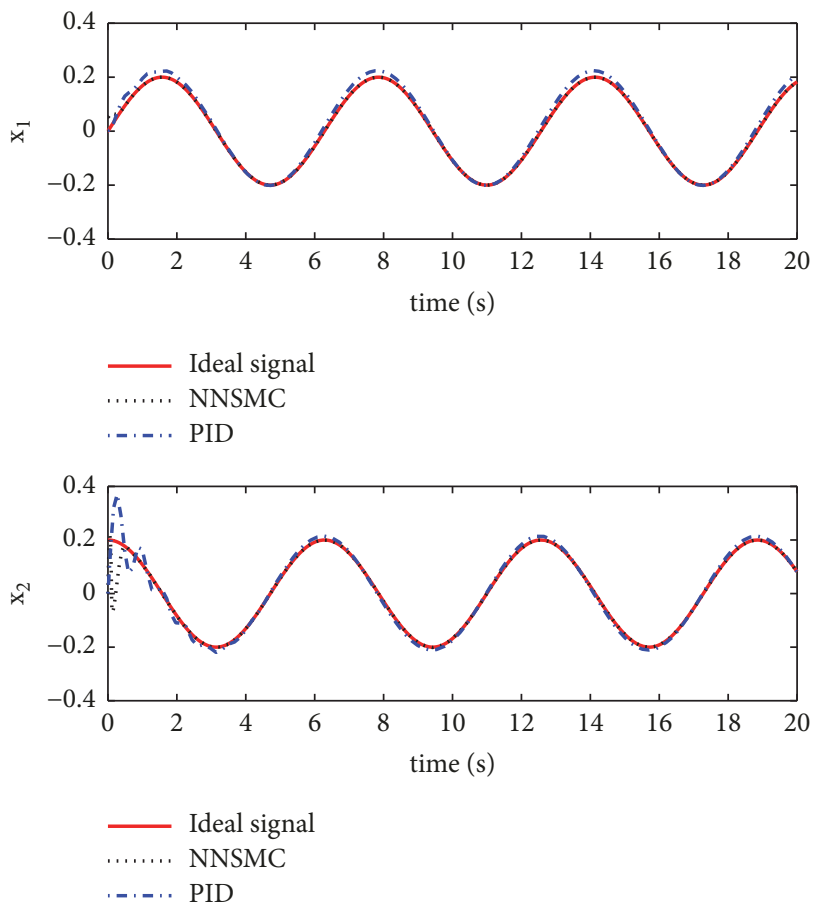

FIGURE 6: The control results of NNSMC versus PID control.

suppressed and the sliding mode quickly reaches the sliding mode manifold.

6.2.2. Case 2. The reference input is chosen as a sinusoidal signal $x_{d}=0.2 \sin (x)$. The controller results and errors are illustrated in Figures 6 and 7. It is shown that the proposed NNSMC can more precisely track the states than the PID controller with shorter dynamic adjustment time from Figure 6. The adjustment time of NNSMC for $x_{2}$ is less than $1 \mathrm{sec}$, but the PID controller adjustment time is more than $3 \mathrm{sec}$. The tracking errors also demonstrate the same conclusion from Figure 7. The mean absolute error 

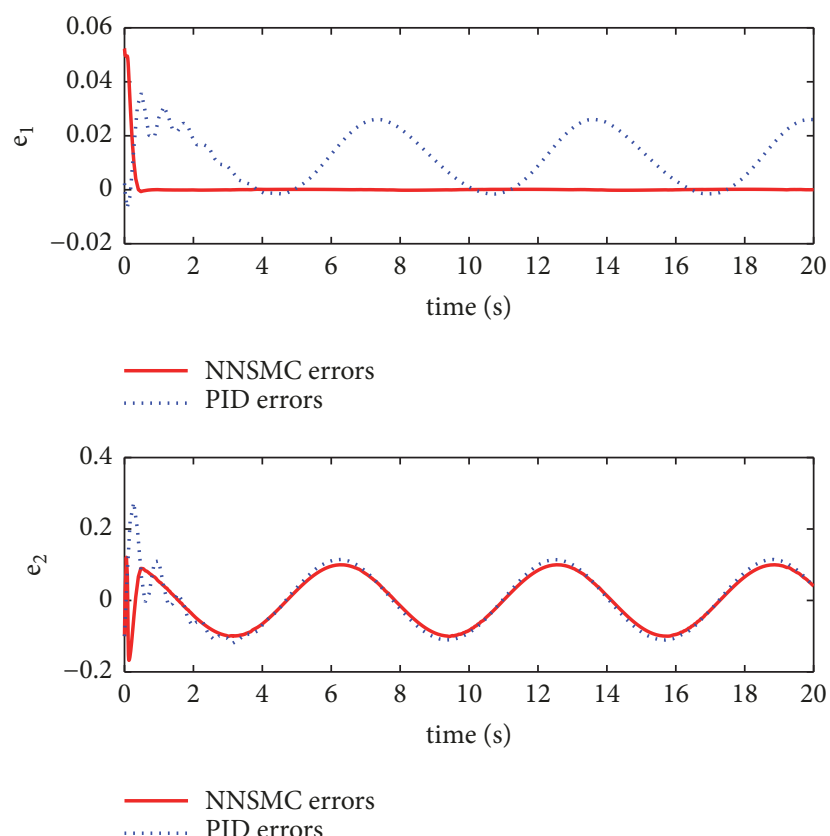

FIgURE 7: The tracking errors of NNSMC versus PID control.

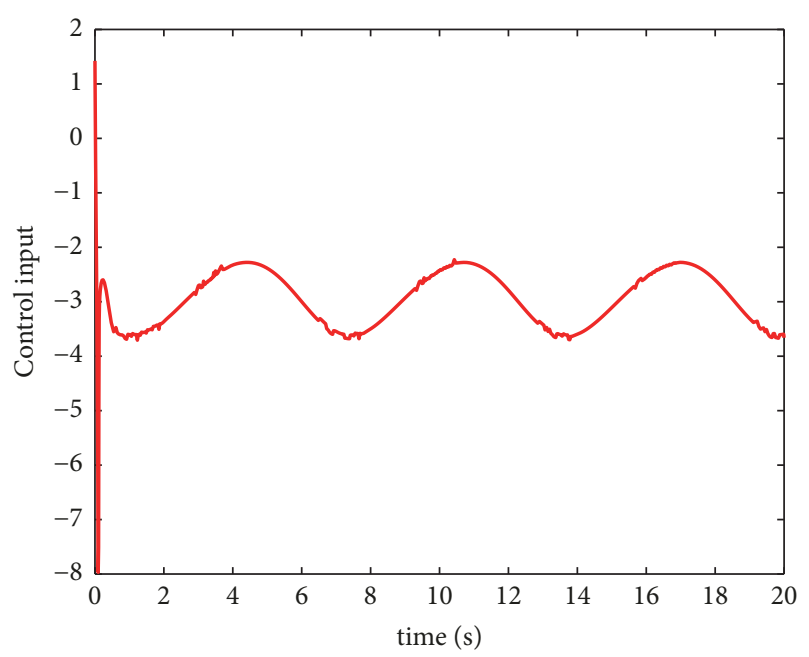

FIGURE 8: The control input of NNSMC.

(MAE) of NNSMC and PID controllers are listed in Table 1. According to the Table 1, the two approaches' MAE of $M A E_{1}$ are $0.0021,0.0121$ and the MAE of $M A E_{2}$ are 0.0066, 0.0078, respectively. It is obviously shown that the proposed NNSMC has better performance for the hysteresis nonlinear system. The NNSMC controller signal is illustrated in Figure 8. From the controller signal, one also finds that the dynamic adjustment time is less than $1 \mathrm{sec}$ in this simulation.

6.2.3. Case 3. In order to demonstrate the performance of the proposed NNSMC, the reference input is selected as $x_{d}=$ $0.1 \sin (x)$ that has a smaller amplitude than Case 1 and the results are illustrated in Figures 9 and 10. The controller input
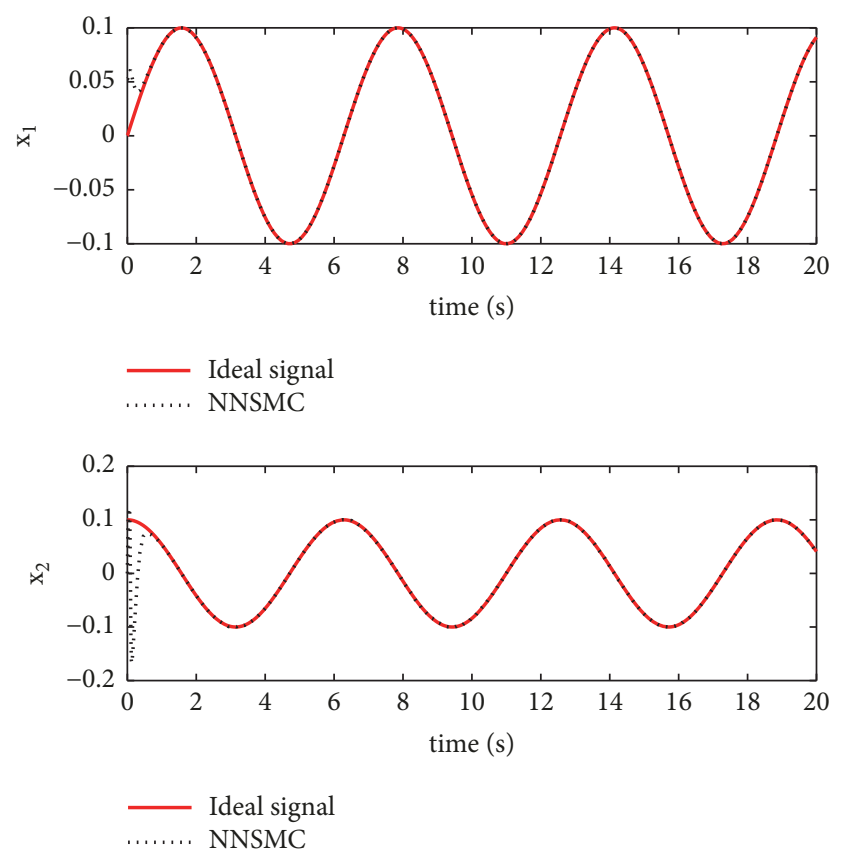

FIGURE 9: The control results of NNSMC with $x_{d}=0.1 \sin (x)$.
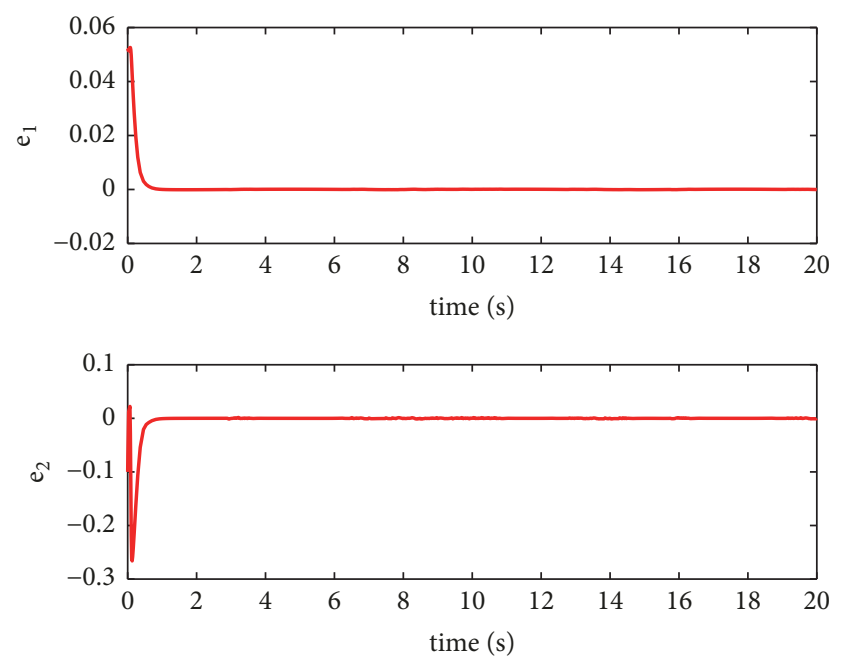

FIGURE 10: The tracking errors of NNSMC with $x_{d}=0.1 \sin (x)$.

TABLE 1: The mean absolute errors of NNSMC and PID controller.

\begin{tabular}{lll}
\hline Controller & $M A E_{1}$ & $M A E_{2}$ \\
\hline NNSMC & 0.0021 & 0.0066 \\
PID & 0.0121 & 0.0078 \\
\hline
\end{tabular}

is shown in Figure 11. It is clearly illustrated that the proposed NNSMC has excellent performance to track the hysteresis nonlinear system. The dynamic adjustment time only has about $0.5 \mathrm{sec}$, then it keeps stable stage and the tracking error holds a neighbourhood of zero. From Figure 10, one can obviously find the same conclusion and the MAE of $M A E_{1}=$ $0.0018, M_{2}=0.0056$, respectively. Compared with the 


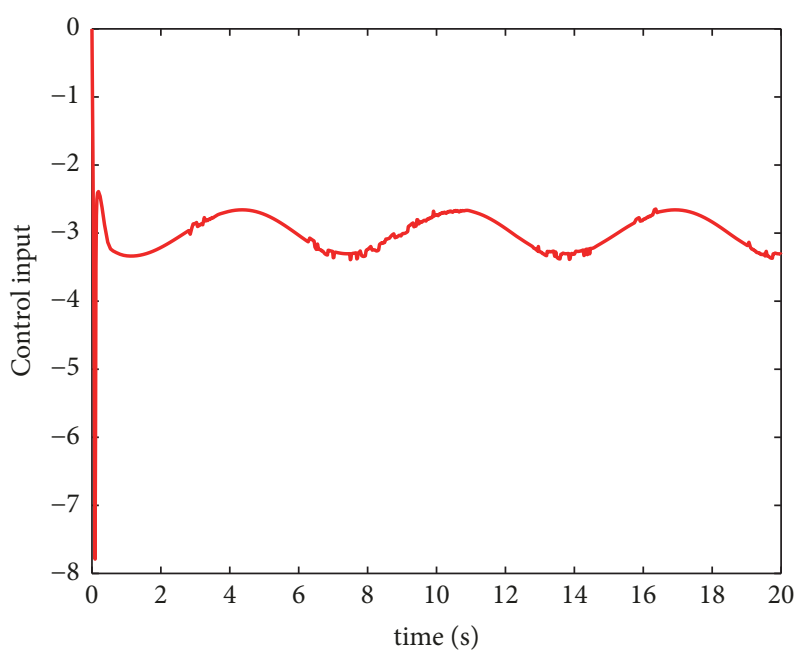

FIGURE 11: The control input of NNSMC with $x_{d}=0.1 \sin (x)$.

results of Case 1, the control precisions are higher on the small signals, that suit the real conditions. With the increase of the reference input signal, the control performance becomes degradation.

\section{Conclusions}

A new neural network identification approach and neural network sliding mode control were proposed for hysteresis nonlinear system with backlash-like model. Since classical adaptive control needs to deal with the tracking error (error transformation or back-stepping, etc.) on the controller design, in this paper a neural network sliding mode controller was designed where it did not need tracking error transformation. Moreover, the unknown system states and the unknown hysteresis section were estimated only by one neural network for saving the computation and simplifying the control design. The stability of the closed loop was guaranteed by Lyapunov theory and the simulation results verified the effectiveness of the presented approaches.

\section{Data Availability}

The data used to support the findings of this study are available from the corresponding author upon request.

\section{Conflicts of Interest}

The authors declare that there are no conflicts of interest.

\section{Acknowledgments}

This work is supported by Shandong Natural Science Foundation of China (ZR2017MF048), the National Natural Science Foundation of China (61803216), and Tai'an Science and Technology Development Program (2017GX0017).

\section{References}

[1] X. Gao, C. Zhang, C. Zhu, and X. Ren, "Identification and control for Hammerstein systems with hysteresis non-linearity," IET Control Theory \& Applications, vol. 9, no. 13, pp. 1935-1947, 2015.

[2] X. Gao, "Adaptive neural control for hysteresis motor driving servo system with Bouc-wen model," Complexity, vol. 2018, Article ID 9765861, 9 pages, 2018.

[3] X. Gao and R. Liu, "Multiscale Chebyshev neural network identification and adaptive control for backlash-like hysteresis system," Complexity, vol. 2018, Article ID 1872493, 9 pages, 2018.

[4] Q. Chen, X. Ren, J. Na, and D. Zheng, "Adaptive robust finitetime neural control of uncertain PMSM servo system with nonlinear dead zone," Neural Computing and Applications, vol. 28, no. 12, pp. 3725-3736, 2017.

[5] Q. Chen, L. Shi, J. Na, X. Ren, and Y. Nan, "Adaptive echo state network control for a class of pure-feedback systems with input and output constraints," Neurocomputing, vol. 275, pp. 13701382, 2018.

[6] J. Na, A. S. Chen, G. Herrmann, R. Burke, and C. Brace, "Vehicle engine torque estimation via unknown input observer and adaptive parameter estimation," IEEE Transactions on Vehicular Technology, vol. 67, no. 1, pp. 409-422, 2018.

[7] S. Wang, J. Na, and X. Ren, "RISE-based asymptotic prescribed performance tracking control of nonlinear servo mechanisms," IEEE Transactions on Systems, Man, and Cybernetics: Systems, vol. 48, no. 12, pp. 2359-2370, 2018.

[8] S. Wang, H. Yu, J. Yu, J. Na, and X. Ren, "Neural-network-based adaptive funnel control for servo mechanisms with unknown dead-zone," IEEE Transactions on Cybernetics, pp. 1-12, 2018.

[9] J. Na, X. Ren, and D. Zheng, "Adaptive control for nonlinear pure-feedback systems with high-order sliding mode observer," IEEE Transactions on Neural Networks and Learning Systems, vol. 24, no. 3, pp. 370-382, 2013.

[10] D. Zheng, W. Xie, X. Ren, and J. Na, "Identification and control for singularly perturbed systems using multitime-scale neural networks," IEEE Transactions on Neural Networks and Learning Systems, vol. 28, no. 2, pp. 321-333, 2017.

[11] Q. Chen, S. Xie, M. Sun, and X. He, "Adaptive non-singular fixed-time attitude stabilization of uncertain spacecraft," IEEE Transactions on Aerospace and Electronic Systems, vol. 54, no. 6, pp. 2937-2950, 2018.

[12] M. Tao, Q. Chen, X. He, and M. Sun, "Adaptive fixed-time fault-tolerant control for rigid spacecraft using a double power reaching law," International Journal of Robust and Nonlinear Control, vol. 29, no. 12, pp. 4022-4040, 2019.

[13] S. H. Chincholkar, W. Jiang, and C. Chan, "A modified hysteresis-modulation-based sliding mode control for improved performance in hybrid DC-DC boost converter," IEEE Transactions on Circuits and Systems II: Express Briefs, vol. 65, no. 11, pp. 1683-1687, 2018.

[14] J. Fang, L. Zhang, Z. Long, and M. Y. Wang, "Fuzzy adaptive sliding mode control for the precision position of piezoactuated nano positioning stage," International Journal of Precision Engineering and Manufacturing, vol. 19, no. 10, pp. 14471456, 2018.

[15] C.-Y. Su, Y. Stepanenko, J. Svoboda, and T. P. Leung, "Robust adaptive control of a class of nonlinear systems with unknown backlash-like hysteresis," IEEE Transactions on Automatic Control, vol. 45, no. 12, pp. 2427-2432, 2000. 
[16] X. Gao, W. Zhao, S. Wang, M. Wang, and X. Ren, "A prescribed performance adaptive control for hysteresis hammerstein system," Journal of Systems Science and Complexity, pp. 1-14, 2018.

[17] J. Na, Q. Chen, X. Ren, and Y. Guo, "Adaptive prescribed performance motion control of servo mechanisms with friction compensation," IEEE Transactions on Industrial Electronics, vol. 61, no. 1, pp. 486-494, 2014. 


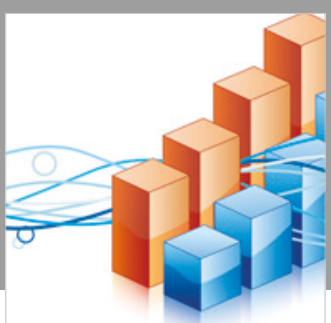

Advances in

Operations Research

\section{-n-m}
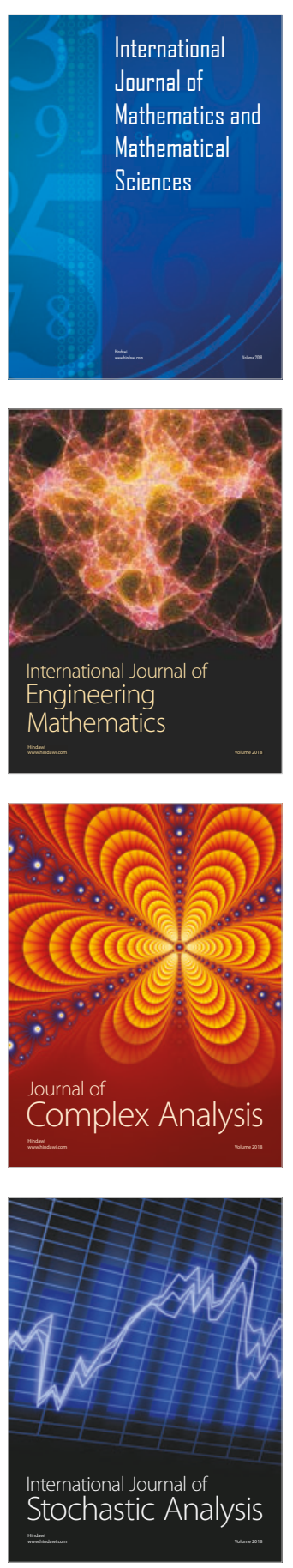
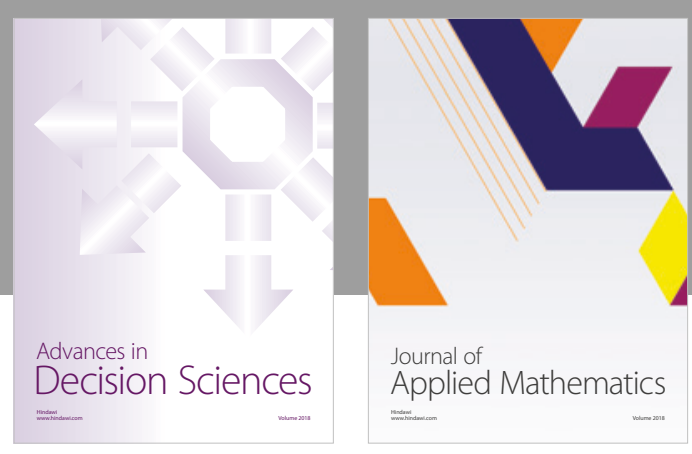

Journal of

Applied Mathematics
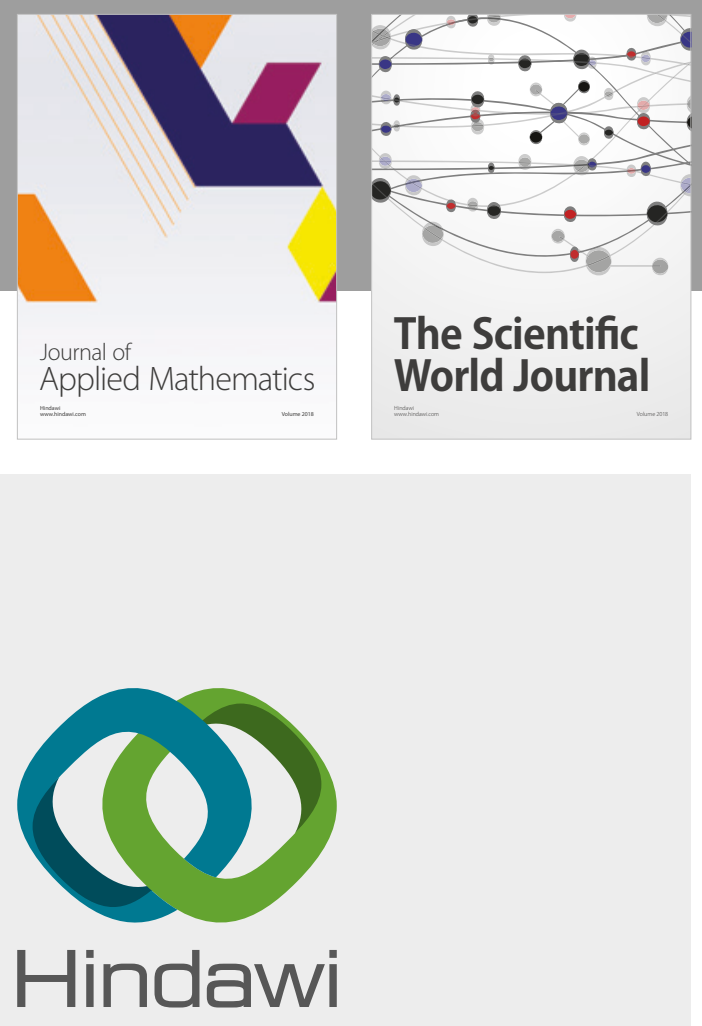

Submit your manuscripts at

www.hindawi.com

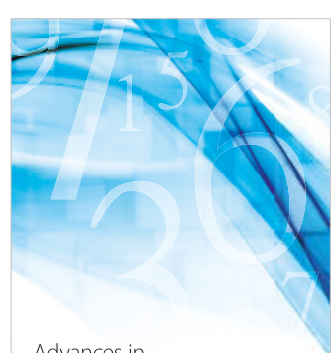

Advances in
Numerical Analysis
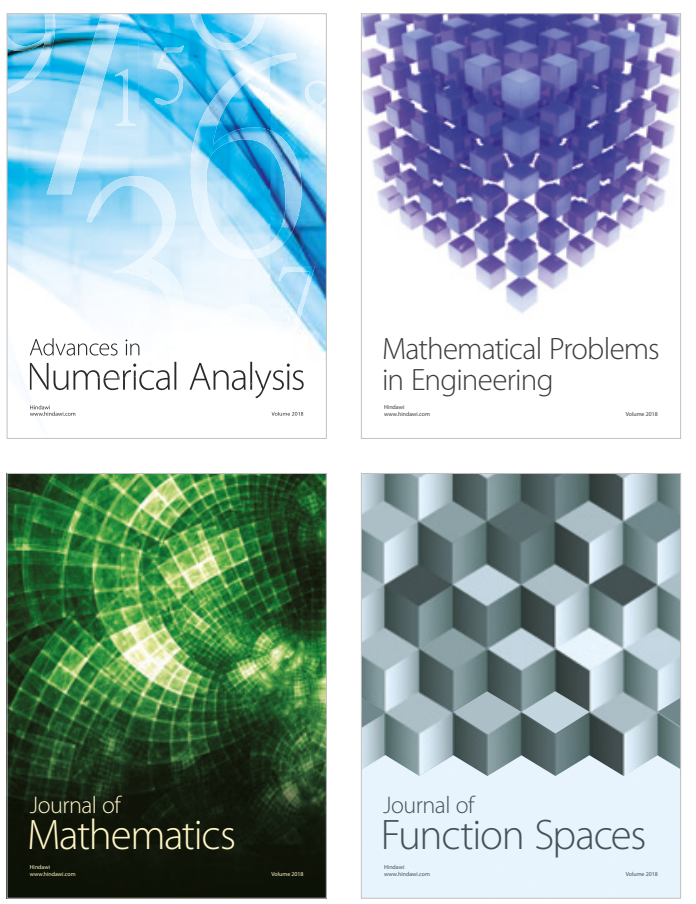

Mathematical Problems in Engineering

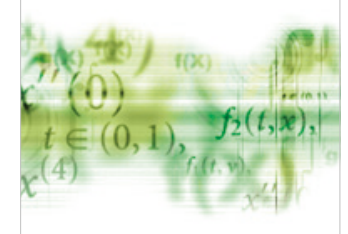

International Journal of

Differential Equations

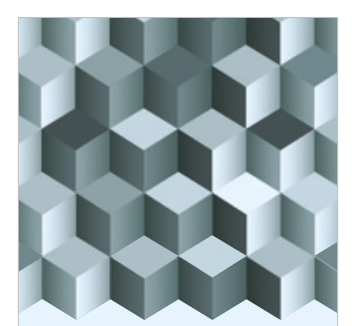

Journal of

Function Spaces
The Scientific

World Journal

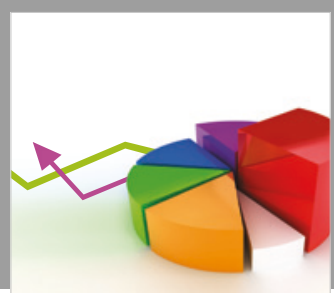

Journal of

Probability and Statistics
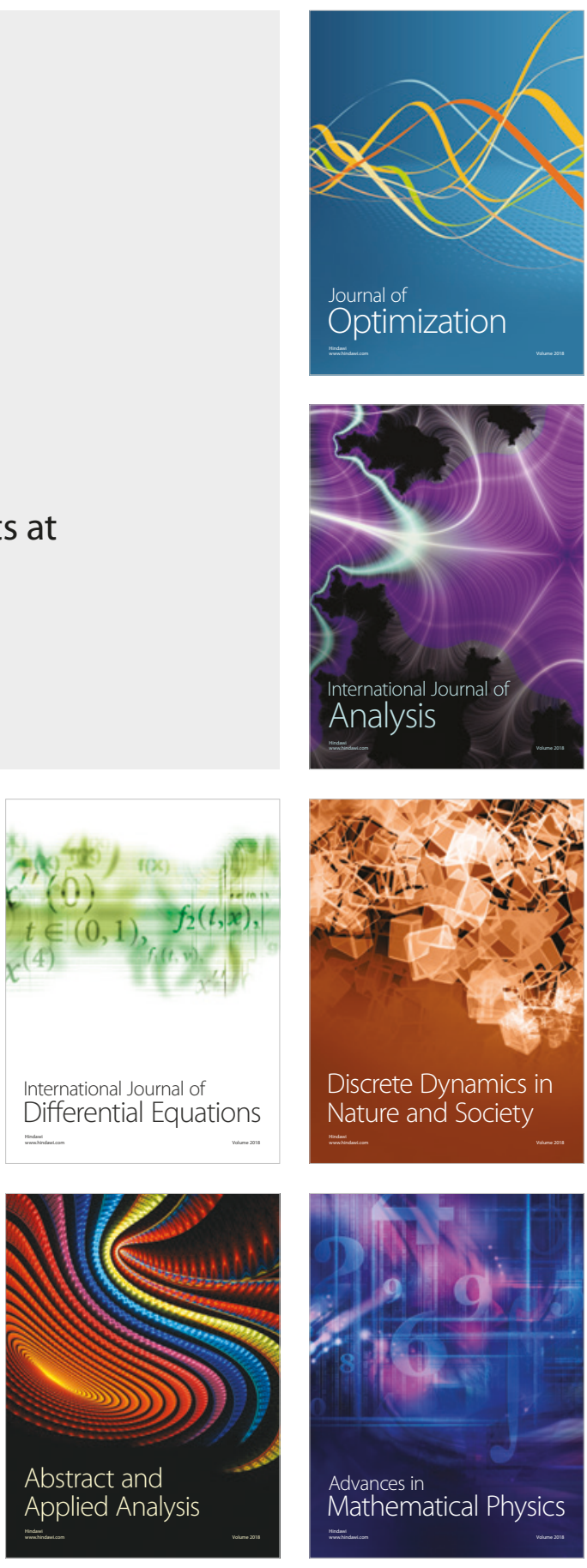